\title{
CONSTRUCCIÓN PARTICIPATIVA DE MODELOS DE NEGOCIOS EN ORGANIZACIONES RURALES
}

\section{Construction of business models in rural organizations}

\section{Carolina Quiñonez Zúñiga* y Luz Elena Laverde Urrea**}

\section{RESUMEN}

El presente artículo expone la herramienta "Modela tu negocio fácil" para la construcción de modelos de negocios participativos en organizaciones rurales, a partir de las características del contexto rural en Colombia. La herramienta es parte de la metodología Runin-Ruta para el éxito de negocios innovadores rurales-, que integra cuatro herramientas que facilitan a las organizaciones su inserción en mercados especializados que demanden valor agregado en sus productos. La fundamentación teórica de la herramienta se cimenta en autores como Osterwalder y Pigneur (2010), Ries (2011), Maurya (2014), Blank y Dorf (2012), quienes plantean los principios de los modelos de negocios que se fundamentan en el Lean Manufacturing japonés, un modelo de gestión que surge a partir de la cultura que adoptaron las empresas japonesas que tenían como objetivo aplicar mejoras en la planta de fabricación a partir de la eficiencia, la rapidez y la agilidad en la toma de decisiones frente a cambios en el mercado. En el diseño metodológico se utilizó el enfoque de la investigación - acción o modo 2, en donde la comunidad además de ser el objeto de investigación, participa e incide en los resultados de acuerdo a sus necesidades, como parte de las conclusiones se resalta la necesidad de realizar procesos de adaptación de metodologías relacionadas con los modelos de negocio que fueron creadas y validadas en contextos de Europa y Estados Unidos; con el propósito de lograr resultados efectivos en el contexto rural colombiano.

Palabras claves: Organizaciones rurales, modelos de negocios, innovación social, enfoque sistémico y participativo.

\footnotetext{
* Comunicadora Social. Magíster en Administración. Docente de la Fundación Universitaria de Popayán y consultora de la Gobernación del Cauca para el Proyecto Construcción de Modelos de Negocios para la Innovación social. Correo electrónico: carolina.quinonez@gmail.com

** Administradora de Empresas, Especialista en Gestión de Proyectos de Desarrollo. Investigadora Principal del Proyecto Construcción de Modelo de Negocios para la Innovación social. Correo electrónico: luzelenalaverde4567@ gmail.com
} 


\begin{abstract}
This article exposes the tool "Model your easy business" for the construction of participatory business models in rural organizations, based on the characteristics of the rural context in Colombia. The tool is part of the Runin-Ruta methodology for the success of innovative rural businesses-, which integrates four tools that make it easier for organizations to enter specialized markets that demand added value in their products. The theoretical foundation of the tool is based on authors such as Osterwalder and Pigneur (2010), Ries (2011), Maurya (2014), Blank and Dorf (2012), who propose the principles of business models that are based on Lean Japanese Manufacturing, a management model that arises from the culture adopted by Japanese companies that aimed to implement improvements in the manufacturing plant based on efficiency, speed and agility in decision-making in the face of changes in the market. In the methodological design the research approach - action or mode 2 was used, in which the community, in addition to being the object of research, participates and influences the results according to their needs, as part of the conclusions the need is highlighted to carry out processes of adaptation of methodologies related to the business models that were created and validated in contexts of Europe and the United States; with the purpose of achieving effective results in the Colombian rural context.
\end{abstract}

Keywords: Rural organizations, business models, social innovation, systemic and participatory approach.

\title{
Introducción
}

Para empezar vale la pena discutir alrededor de los factores que inciden en el desempeño organizacional en el contexto rural, al respecto es necesario tener en cuenta que:

"La eficacia de las organizaciones de productores a menudo se ve limitada por las restricciones legales, la escasa capacidad de gestión, el acaparamiento de las organizaciones por las élites, la exclusión de los pobres y la falta de reconocimiento de su calidad de socios plenos por parte del Estado " (Vargas \& Montoya, 2011, p.12).

Conforme a estudios de caso sobre la comercialización de productos y servicios de organizaciones microempresariales rurales ejecutados por el Instituto Interamericano de Cooperación para la Agricultura (IICA), se identificaron limitaciones principalmente en cinco aspectos: a) bajos volúmenes de producción, b) inadecuada 
presentación de los productos, especialmente en lo relacionado con empaques y etiquetas, c) deficiencias en la calidad e inocuidad, especialmente de los productos frescos; d) poco rigor en el cálculo de los costos de producción y e) ausencia de mecanismos apropiados para la promoción de los productos. Se evidenció además que

"Los escasos recursos de las asociaciones impiden incrementar sus volúmenes de producción y acceder a mejores mercados. También existe poco control en los costos de producción y en el cálculo de precios de venta, escasa estandarización de procesos y productos, y poca exigencia de criterios de calidad en los mercados locales que limita el ingreso a otros mercado" (Rodriguez \& Riveros, 2016, p.31).

En síntesis las organizaciones de productores rurales en Colombia deben enfrentar exigentes retos para acceder directamente y en condiciones equitativas a mercados formales que impliquen ventajas de negociación. Si bien estas organizaciones cuentan con conocimientos y experiencia sobre los mecanismos para comercializar en mercados informales, desconocen aspectos que les facilitaría su articulación directa con el mercado, el aprovechamiento de los apoyos institucionales disponibles y el mejoramiento de su capacidad de gestión comercial a través de modelos de negociación innovador e inclusivo, económicamente rentable y con mercados mejor remunerados.

En este sentido, según López y Castrillón (2007) cuando se crean condiciones apropiadas, los pequeños productores pueden realizar transformaciones productivas y mejoramientos tecnológicos que incrementen notoriamente sus ingresos, dado que pueden proyectarse en mercados amplios. Un modelo de negocio bien concebido contribuye a que la organización rural sea sostenible en el tiempo, por medio de procesos de agregación de valor, dentro de una sólida organización socioeconómica y la adopción de tecnología apropiada.

Este artículo, presenta en primer lugar los fundamentos teóricos alrededor de los modelos de negocios, la organización rural y la innovación social. En segundo lugar, la metodología que guió el desarrollo del trabajo de investigación. En tercer lugar, se relacionan los resultados de la implementación de la herramienta "Modela tu negocio fácil" para la construcción de modelos de negocios en organizaciones rurales, bajo un 
enfoque sistémico y participativo, que facilita la inserción de las organizaciones en mercados especializados mejor remunerados. Para finalizar se presentan las conclusiones alrededor de la necesidad de abordar de manera más eficiente la elaboración de un modelo de negocio en organizaciones rurales y su aporte a la construcción de innovación social.

\section{Marco teórico y conceptual}

Los modelos de negocios más representativos encontrados en la revisión literaria sugieren que están constituidos a través de bloques y esquemas, los cuales representan segmentos y características comunes para identificar componentes útiles en una cadena de valor y en segmento de mercado establecido. El concepto Modelo de Negocios es acuñado por Osterwalder y Pigneur (2010), para estos autores:

[...] un modelo de negocio describe la lógica sobre cómo una organización crea, captura y entrega valor". Los modelos de negocios tratan básicamente de las maneras específicas en las cuales se lleva a cabo un negocio, lo que significa que es la forma como la empresa genera ingresos gracias al posicionamiento de una marca en un nicho del mercado. (p.4)

Desde el planteamiento Osterwalder y Pigneur (2010), son diferentes los abordajes y adaptaciones que se han dado al concepto Modelo de Negocios--Bussines Models-, a continuación se relacionan cronológicamente los abordajes que han construido alrededor de este concepto. Para ello, se realizó una búsqueda en las bases de datos de EBSCO, Google Académico, y Scielo. Como resultado se analizaron los libros de siete autores de mayor citación (Alexander Osterwalder, Eric Ries, Ash Maurya, Steve Blank, Bob Dorf, Yves Pigneur) quienes han realizado aportes para la estructuración y afinación del acervo teórico de los modelos de negocios para organizaciones donde el activo principal es el conocimiento. (Tabla 1). 
Tabla 1. Abordaje cronológico de los Modelos de Negocios: 2010-2017

\begin{tabular}{|c|c|c|}
\hline $\begin{array}{c}\text { Marco } \\
\text { conceptual }\end{array}$ & Descripción & Autores \\
\hline $\begin{array}{c}\text { Business Model } \\
\text { Generation }\end{array}$ & $\begin{array}{l}\text { Describe los elementos para estructurar un } \\
\text { modelo de negocio en nueve componentes. Se } \\
\text { fundamenta en la propuesta de valor como eje de } \\
\text { innovación y catalizador de los demás } \\
\text { componentes en una estrategia de ingreso a } \\
\text { mercados complejos por la vía de productos } \\
\text { innovadores. Proporciona herramientas prácticas } \\
\text { para comprender, diseñar e implementar un } \\
\text { nuevo modelo de negocio o renovar uno antiguo. } \\
\text { Cómo elemento distintivo se destaca que el libro } \\
\text { fue cocreado por } 470 \text { profesionales de } 45 \text { países. }\end{array}$ & $\begin{array}{c}\text { (Osterwalder \& } \\
\text { Pigneur, 2010) }\end{array}$ \\
\hline $\begin{array}{c}\text { El método lean } \\
\text { startup }\end{array}$ & $\begin{array}{l}\text { Se establece la actividad emprendedora y la } \\
\text { innovación sobre una base científica. El } \\
\text { movimiento del método Lean Startup pretende } \\
\text { garantizar que quienes crearán el siguiente gran } \\
\text { producto tengan las herramientas necesarias. } \\
\text { El método Lean Startup, está diseñado para } \\
\text { enseñar a gestionar una empresa de base } \\
\text { tecnológica a través de la experimentación. En } \\
\text { lugar de hacer planes complejos basados en } \\
\text { muchas suposiciones, se pueden hacer ajustes } \\
\text { constantes bajo el enfoque: Crear-Medir- } \\
\text { Aprender, que es el núcleo central de este } \\
\text { método. A través de este proceso de dirección, es } \\
\text { posible saber si ha llegado el momento de hacer } \\
\text { un giro drástico llamado pivote o si la empresa } \\
\text { debe perseverar en su trayectoria actual. }\end{array}$ & (Ries, 2011) \\
\hline Running Lean & $\begin{array}{l}\text { El mayor aporte que hace el autor es la creación } \\
\text { del Lean Canvas, una adaptación del modelo de } \\
\text { negocio CANVAS propuesto inicialmente por } \\
\text { Osterwalder y Pigneur (2010). } \\
\text { El libro sigue el proceso típico de metodologías } \\
\text { lean: Estructurar modelo de negocio - Testeo de } \\
\text { hipótesis - Producto viable mínimo, pero lo } \\
\text { plantea de una manera práctica dando } \\
\text { información operativa que se puede aplicar } \\
\text { directamente a un proyecto específico. } \\
\text { La estructura del libro comprende } 2 \text { bloques: } \\
\text { a. Conceptualización del modelo de negocio a } \\
\text { través del Lean Canvas. } \\
\text { b. Experimentación para afinar el modelo de } \\
\text { negocio, a través de entrevistas a clientes. }\end{array}$ & (Maurya, 2014) \\
\hline
\end{tabular}




\begin{tabular}{|c|c|c|}
\hline $\begin{array}{l}\text { The Startup } \\
\text { Owner's Manual: } \\
\text { The Step-by-Step } \\
\text { Guide for } \\
\text { Building a Great } \\
\text { Company }\end{array}$ & $\begin{array}{l}\text { Los autores presentan consejos, técnicas y } \\
\text { mejores prácticas para la construcción de } \\
\text { empresas de base tecnológica exitosa. Se divide } \\
\text { en tres bloques: } \\
\text { a. La Guía de Estrategia, proporciona } \\
\text { antecedentes y una visión general del Modelo } \\
\text { de Negocio y procesos de desarrollo de } \\
\text { clientes } \\
\text { b. Web / móvil, proporciona todos los detalles } \\
\text { del proceso necesario para crear una empresa } \\
\text { de base tecnológica sólida y sostenible cuya } \\
\text { estrategia comercial se fundamente en uso } \\
\text { intensivo de canales digitales } \\
\text { c. Canales Físicos, proporciona todos los } \\
\text { detalles del proceso necesario para crear una } \\
\text { empresa de base tecnológica sólida y } \\
\text { sostenible cuya estrategia comercial se } \\
\text { fundamente en uso intensivo de canales } \\
\text { tradicionales (radio, prensa, TV e impresos). }\end{array}$ & $\begin{array}{c}\text { (Blank \& Dorf, } \\
\text { 2012) }\end{array}$ \\
\hline $\begin{array}{c}\text { The four steps to } \\
\text { the epiphany }\end{array}$ & $\begin{array}{l}\text { El libro constituye una metodología precursora de } \\
\text { Lean Startup enfatizando en el desarrollo de la } \\
\text { empresa y proyecto orientado totalmente al } \\
\text { cliente, basando su lógica en la gran } \\
\text { incertidumbre con la que tienen que lidiar los } \\
\text { emprendedores de muy diversos sectores. } \\
\text { Según el autor, después de la lectura del Business } \\
\text { model generation de Osterwalder y Pigneur } \\
\text { (2010), esta perspectiva ofrece una serie de } \\
\text { conocimientos complementarios de gran valor al } \\
\text { momento de implementar la metodología, aunque } \\
\text { no se llegue finalmente a aplicar. } \\
\text { Más allá del conjunto que supone la metodología } \\
\text { de Desarrollo de clientes -Customer } \\
\text { Development-, el libro desarrolla elementos de } \\
\text { interés como la curva de adopción del ciclo de } \\
\text { vida de tecnología, los evengelizadores -early } \\
\text { adopters-, los abismos, nichos de mercado } \\
\text { especializados, el aprendizaje basado en la } \\
\text { dualidad de hipótesis sobre el problema y } \\
\text { solución, entre otros que intentan diferenciar la } \\
\text { perspectiva de Producto Vs el nuevo enfoque } \\
\text { hacia el cliente. }\end{array}$ & (Blank, 2013) \\
\hline
\end{tabular}




\begin{tabular}{|c|c|c|}
\hline $\begin{array}{c}\text { Value proposition } \\
\text { design }\end{array}$ & $\begin{array}{l}\text { Es un libro gráfico, Value Proposition Design } \\
\text { simplifica las ideas complejas en ilustraciones } \\
\text { rápidamente legibles con solo los detalles más } \\
\text { prácticos e importantes para la identificación y } \\
\text { estructuración de la Propuesta de Valor como } \\
\text { elemento nuclear en los modelos de negocio. } \\
\text { Establece una diferenciación en el CANVAS, } \\
\text { por un lado el Perfil del Cliente, que tiene como } \\
\text { propósito clarificar y conocer a profundidad el } \\
\text { cliente, y por otro, el Mapa de Valor, que tiene } \\
\text { como fin la descripción detallada de los } \\
\text { mecanismos para crear valor a dicho cliente. La } \\
\text { meta radica en lograr encaje o match entre estas } \\
\text { dos perspectivas. }\end{array}$ & $\begin{array}{l}\text { (Osterwalder.,et } \\
\text { al, 2014) }\end{array}$ \\
\hline $\begin{array}{l}\text { Scaling Lean: } \\
\text { Mastering the } \\
\text { Key Metrics for } \\
\text { Startup Growth }\end{array}$ & $\begin{array}{l}\text { El libro enfatiza en las métricas que definen un } \\
\text { modelo de negocios eficaz, a partir de dichas } \\
\text { métricas, se proponen estrategias de crecimiento } \\
\text { e inversión para la gestión e interacción con } \\
\text { diferentes stakeholders tanto internos como } \\
\text { externos. El libro surge a partir de las } \\
\text { dificultades para implementar el Modelo de } \\
\text { Negocio cuando las empresas crecen y se tornan } \\
\text { complejas. } \\
\text { Para el autor, el modelo CANVAS le da enfoque } \\
\text { valioso a la empresa al desentrañar los entramados } \\
\text { de solución de problema, como el enfoque } \\
\text { Producto-Mercado. Pero plantea como } \\
\text { interrogante ¿Qué tan útiles son el CANVAS y el } \\
\text { LEAN CANVAS como herramientas para la } \\
\text { planificación estratégica y la gestión del } \\
\text { rendimiento? . En el libro el autor explica la } \\
\text { utilidad de las herramientas para modelar el éxito } \\
\text { de la puesta en marcha. En esencia, un CANVAS } \\
\text { o LEAN CANVAS es una estrategia de gestión } \\
\text { estratégica y de despliegue para desarrollar } \\
\text { nuevos modelos comerciales (o re-estructurar } \\
\text { perfiles existentes). }\end{array}$ & (Maurya, 2016) \\
\hline
\end{tabular}




\begin{tabular}{|c|c|c|}
\hline The Startup Way, & $\begin{array}{l}\text { En este libro, los principios de los Modelos de } \\
\text { Negocios migran hacia la cultura organizacional } \\
\text { bajo la premisa de hacer del emprendimiento una } \\
\text { disciplina fundamental de toda empresa, inserta } \\
\text { en su ADN (en términos de pro actividad, } \\
\text { creatividad, actitud crítica y visión de mercado) } \\
\text { Para el autor, la forma de mantenerse vigente en } \\
\text { los mercados se puede sintetizar en dos cosas: } \\
\text { tratar a los empleados como clientes y tratar a las } \\
\text { unidades de negocio como las Empresas de Base } \\
\text { Tecnológica. } \\
\text { Para lograrlo, es necesario adaptar las } \\
\text { metodologías de gestión de los modelos de } \\
\text { negocios a las áreas funcionales de la propia } \\
\text { organización, promoviendo paralelamente el } \\
\text { pensamiento creativo y el espíritu emprendedor } \\
\text { en el talento humano }\end{array}$ & (Ries, 2017) \\
\hline
\end{tabular}

Fuente: Elaboración propia.

Ahora bien, para esta investigación es necesario considerar la influencia del modelo de negocio en organizaciones, para ello es preciso traer a colación el concepto de Piñeiro (1985) alrededor de este tema

[...] Las organizaciones rurales son grupos dotados de procedimientos formalmente establecidos. Son conjuntos sociales considerados, en primer lugar, desde el ángulo de su estructura ya que toda organización implica un modelo más o menos jerárquico y constituye una asociación con vistas a la realización de objetivos comunes. (p.4)

En virtud de lo anterior varias entidades y proyectos, que apoyan el desarrollo rural en Colombia, han emprendido iniciativas en la formación de capacidades organizativas, decisivas para la conformación de líderes empresariales y organizaciones, que hoy promueven o consolidan su inserción estable a los mercados y sobresalen en desempeño, generación de oportunidades, la capacidad de relacionamiento, así como en el mejoramiento social y económico. En ese sentido, es importante considerar la innovación social, como otro concepto fundamental para esta investigación. Para, Morales (2009) se entiende como: 
[...] un proceso que involucra la relación de las gentes que trabajan de manera cohesionada para resolver problemas propios de la comunidad, se hace preciso hablar aquí sobre las dinámicas organizacionales que soportan dichos vínculos La transformación social tiene que ver con un cambio exigido por las personas o la sociedad, es un cambio de la gente para la gente, un cambio que se define así mismo en un contexto de injusticia, de desigualdades, de falta de recursos y acceso a los mismos (p.152).

Con respecto a lo anterior los procesos de innovación social le han permitido a las comunidades en especial a las organizaciones rurales ampliar sus capacidades, como en el caso de la habilidad de liderazgo desde lo local y participativo, permitiendo la construcción de capital social, al punto de que sus miembros se logran movilizar de manera conjunta en la toma de decisiones, el empoderamiento de los individuos, la reconstrucción del territorio y la construcción de visiones de futuro compartidas para promover iniciativas que faciliten resolver problemáticas locales.

En consecuencia, la transformación social desde la perspectiva de Landazury y Ferrer (2016), tiene que ver con un cambio exigido por las personas o la sociedad, es un cambio de la gente para la gente, un cambio que se dé así mismo en un contexto de injusticia, de desigualdades, de falta de recursos y acceso a los mismos.

Los abordajes teóricos antes mencionados sirven de sustrato para la estructuración de un modelo de negocio que mantenga los principios de las metodologías que propenden por un acercamiento efectivo de las organizaciones al mercado, a la vez, que posibilitan el atemperamiento de estos instrumentos a las realidades de las organizaciones rurales en ambientes como el del departamento del Cauca en Colombia. Con la revisión literaria antes mencionada, el ejercicio de investigación avanzó hacia la fase de trabajo de campo para la construcción de la herramienta. 


\section{Metodología}

En esta investigación se utilizó el enfoque - acción o modo 2, en donde la comunidad además de ser el objeto de investigación, participa e incide en los resultados de acuerdo a sus necesidades e intereses, asumiendo de esta manera un papel activo en el proceso de investigación.

En el diseño metodológico se trabajó con cinco unidades de análisis conformada por organizaciones rurales del departamento del Cauca Corseda, Apropesca, Asorcafé, Arcauca y Rescate Cacaotero, pertenecientes a las cadenas productivas de la seda, piscicultura, cafés especiales, yuca y cacao respectivamente en el departamento del Cauca, en sus procesos de identificación y validación de sus modelos de negocios. Las organizaciones fueron seleccionadas como una muestra representativa de las agrocadenas priorizadas en diferentes ejercicios de planificación territorial en el departamento del Cuaca como el Plan Regional de Competitividad y el Plan Departamental de Desarrollo.

Tabla 2: trabajo de campo realizado

\begin{tabular}{|l|l|l|l|}
\hline Organización & $\begin{array}{l}\text { Personas } \\
\text { participantes }\end{array}$ & Talleres & $\begin{array}{l}\text { Entrevistas } \\
\text { realizadas }\end{array}$ \\
\hline Corseda, & 50 & 8 & 50 \\
\hline Apropesca, & 50 & 8 & 50 \\
\hline Asorcafé, & 70 & 8 & 70 \\
\hline Arcauca & 20 & 8 & 20 \\
\hline $\begin{array}{l}\text { Rescate } \\
\text { Cacaotero }\end{array}$ & 30 & 8 & 30 \\
\hline
\end{tabular}

Fuente: Elaboración propia

Las organizaciones en mención representan cadenas productivas distintas y muestran niveles de madurez diferentes respecto a su estructura organizativa, su capacidad productiva y de gestión, y su relacionamiento con el mercado, condiciones que -para efectos de la investigación- permitieron trabajar en diferentes escenarios y 
analizar las dificultades de estas organizaciones en su articulación con mercados diferenciados.

Las herramientas utilizadas para toma de información primaria fueron: talleres, entrevistas y visitas a las organizaciones objeto de estudio. Los talleres permitieron la interacción de los actores en forma colectiva, a través de estos es posible identificar las necesidades puntuales de los actores involucrados.

Las entrevistas y visitas facilitaron la comprensión de la visión del mundo que poseen los actores sociales y complementan la información obtenida a través de los talleres, ya que generalmente dicha información no se encuentra registrada y hace parte del saber y las dinámicas propias de los actores participantes. Tanto los talleres como las entrevistas propiciaron que las personas hablen sobre lo que saben, piensan y creen para generar una interacción de los conocimientos que poseen con los que reciben.

\section{Resultados.}

El trabajo de campo desarrollado en el marco de esta investigación con Organizaciones rurales, permitió identificar mejores prácticas de acuerdo a las dinámicas del contexto para la construcción y validación de una metodología que facilita la renovación de los modelos de negocio de las empresas asociativas rurales. Si bien se tomó como punto de partida el acervo teórico alrededor de los modelos de negocio; sobre el trabajo en terreno sólo se presenta el diseño resultante en virtud del espacio disponible en este artículo, y se destaca que posibilitó la adaptación e incrementó los niveles de pertinencia hasta llegar a la construcción de la Metodología Runin -Ruta Para el Éxito de Negocios Innovadores Rurales-, integrada por cuatro (4) herramientas que facilitan la construcción de modelos de negocios participativos en este tipo de organización, a través de una ruta que las lleva hacia el reconocimiento de su estado actual, la identificación de una nueva propuesta de modelo de negocio, la planeación para el desarrollo del modelo de negocio propuesto, y finaliza con el monitoreo y seguimiento del mismo. 
La Ruta para el Éxito de Negocios Innovadores Rurales, RUNIN, es una caja de herramientas para la construcción de modelos de negocios participativos en organizaciones rurales, integrada por 4 herramientas que permiten:

I. Evalúa Fácil, realiza El diagnóstico de la organización y la identificación de su modelo de negocio actual.

II. Modela tu negocio fácil, elabora un nuevo modelo de negocio para que la organización rural haga más efectiva su relación con el cliente y acceda a mercados diferenciados y mejor remunerados, bajo un enfoque sistémico y participativo.

III. Planea fácil, facilita la elaboración de un plan de acción que facilite la priorización de actividades para la implementación del modelo de negocio.

IV. Monitorea fácil, hace posible el seguimiento de las metas establecidas en el desarrollo del modelo de negocio propuesto, para determinar la viabilidad y éxito del modelo.

De esta manera, RUNIN presenta una ruta para identificar con mayor claridad las necesidades de la organización rural y establecer propuestas de valor a sus productos, a partir de las capacidades de gestión en sus diferentes áreas institucionales.

Gráfico 1. Metodología Runin

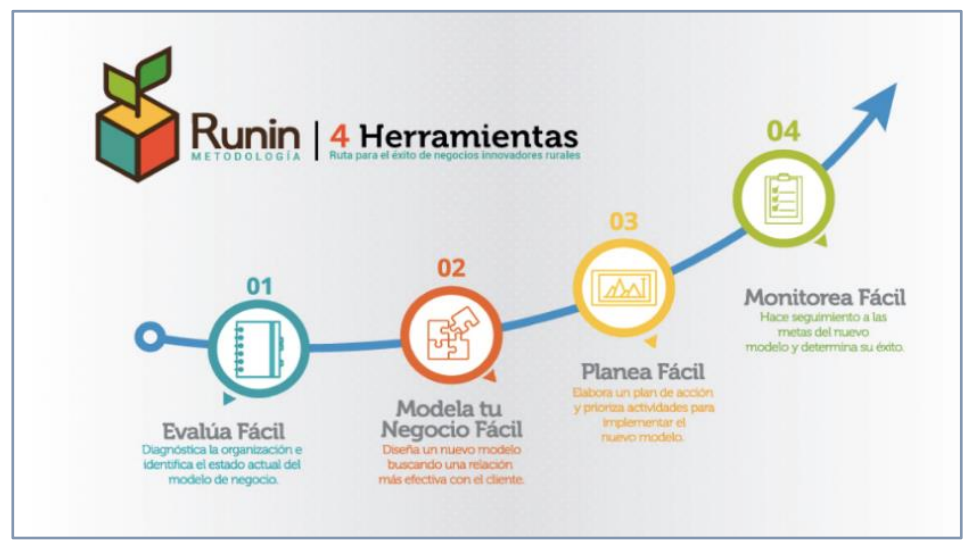

Fuente: elaboración propia 
Construcción participativa de modelos de negocios en organizaciones rurales

Se destaca de esta metodología la concepción de sus cuatro herramientas bajo un enfoque sistémico y participativo, que propende por la inclusión de los integrantes de la Organización en su desarrollo y que permite concebir un modelo de negocio a partir de un mejor reconocimiento del entorno y del mercado, y de las capacidades técnicas y de gestión de las organizaciones rurales.

Aunque la metodología considera el uso de las cuatro herramientas para el desarrollo de un proceso efectivo de las organizaciones rurales en mercados mejor remunerados, para efectos de investigación se abordará en detalle los componentes de la herramienta 2 "Modela de tu negocio fácil" y los factores diferenciales frente a las propuestas de modelos de negocios tradicionales.

\section{Herramienta 2 "Modela tu negocio Fácil"}

Figura 1. Propuesta Modelo de negocios para organizaciones productivas rurales

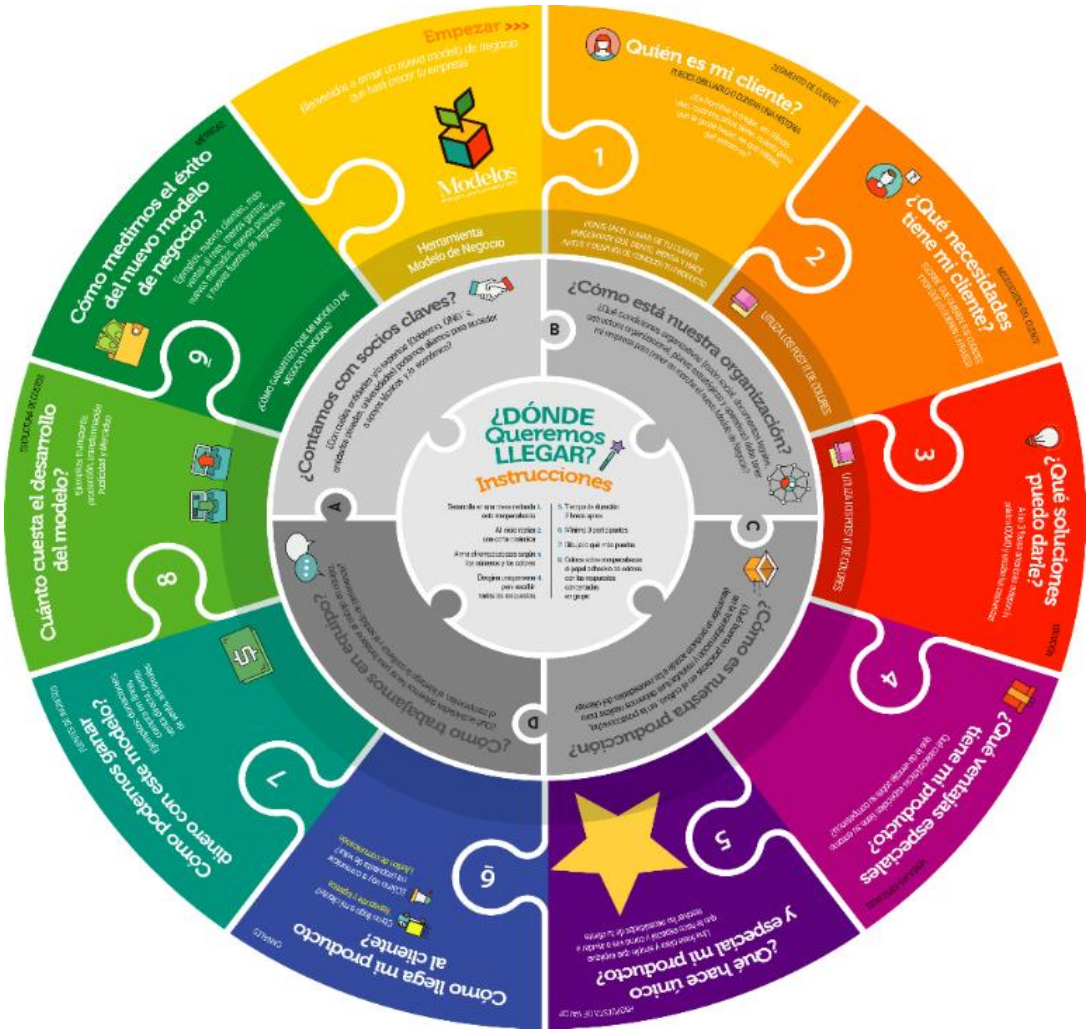

Fuente: Metodología Runin- elaboración propia. 
La herramienta "Modela tu negocio fácil", permite la elaboración de modelos de negocio, reduciendo la incertidumbre y abordando oportunidades detectadas en mercados mejor remunerados, según sus propias capacidades y las tendencias de mercado. Brinda componentes de análisis que le permiten mayor acercamiento y conocimiento de sus clientes, y de las problemáticas que enfrenta (que pueden ser resueltas con la nueva propuesta de modelo de negocio).

Esta herramienta contiene un rompecabezas de trece (13) fichas, donde cada una corresponde a los componentes para la elaboración del nuevo modelo de negocio, basado en los 9 componentes del modelo Lean Canvas de Eric Ries (2011): Segmento de Clientes, Problema, Solución, Proposición Única de Valor, Canales, Flujo de Ingresos, Estructura de Costos, Métricas Claves y Ventaja Especial.

A partir del ejercicio de investigación con cinco organizaciones rurales y el trabajo de campo realizado con las mismas, se identificaron algunos elementos que fue necesario considerar a la hora de plantear una propuesta de Modelo de Negocio para Organizaciones Rurales.

\section{Elementos diferenciadores}

Secuencia de los componentes del modelo. El trabajo de campo realizado con los productores rurales evidenció, que la ubicación original de los componentes planteada desde la versión original de Osterwalder y Pigneur (2010), es de difícil comprensión para los productores rurales. No seguir una secuencia en la ubicación de los componentes del lienzo, causaba confusión y frustración entre los miembros de organización rural en la realización de su Modelo de Negocio, a quienes les tomaba mucho tiempo ubicarse en cada componente para continuar con el ejercicio. Por ello, la herramienta planteada si bien conserva los componentes originales del Lean Canvas, los plantea de manera secuencial, de tal forma que el productor rural identifica con claridad el ciclo que debe desarrollar para abordar su modelo de negocio. 
Adaptación del lenguaje al contexto de los productores rurales. Si bien el éxito de la propuesta de Osterwalder y Pigneur (2010), radica en la capacidad de síntesis para plasmar en un lienzo, la forma en como la organización puede lograr penetrar con éxito en el mercado, en el caso de las organizaciones rurales, es necesario replantear la forma de las preguntas que orientan el desarrollo de cada componente e incluir ejemplos que les permita comprender el propósito de la información que requiere cada campo del modelo de negocio. En este proceso es fundamental establecer un lenguaje común que facilitara la comprensión de la herramienta.

\section{Incorporación de los componentes transversales como condiciones}

\section{habilitantes para que una organización rural desarrolle su modelo de negocio.}

Diferentes estudios y ejercicios de diagnóstico en el ámbito territorial coinciden en es necesario tener en cuenta factores críticos, que constituyen la base o plataforma sobre la cual la organización rural necesita trabajar para desplegar su modelo de negocio. Desde la perspectiva de este trabajo se plantea la incorporación de componentes como: i) organizacional, ii) asociatividad, iii) trabajo en equipo y iv) productividad, que si bien hace parte de la estructura interna de la organización, no se pueden desligar de una propuesta de modelos de negocios en organizaciones rurales, pues es común que este tipo de organizaciones presenten dificultades en estos componentes que de no ser atendidas oportunamente, fracasarían cualquier intento de generar un modelo de negocio efectivo.

Enfoque sistémico: en el trabajo realizado con las organizaciones rurales es de vital importancia la interacción entre los miembros de la organización alrededor de un objetivo común, facilita la participación de los diferentes actores, haciendo posible que todas las partes que intervienen se interconectan y se relacionan entre sí, permitiendo un intercambio de conocimiento, saberes, aprendizaje. Con la aplicación del modelo de negocio, se pudo evidenciar la importancia de que el flujo de información, intercambio de experiencias, se de en igualdad en condiciones.

Los elementos teóricos de referencia en los que se fundamenta el modelo provienen principalmente de la teoría de los sistemas (Bertalanfy, 1976). De la teoría general de 
los sistemas incorpora la definición de sistema y su aplicación a la intervención social. El desarrollo de la teoría sistémica es atribuido a Ludwig Von Bertalanffy, un biólogo que definió los sistemas como elementos que interactúan unos con otros.

En enfoque sistémico en la herramienta, se relaciona con la propuesta circular, a manera de rompecabezas en donde la totalidad de la herramienta depende los todos los elementos interconectados entre si, el rompecabezas se puede armar sobre una superficie plana, facilitando la ubicación en mesa redonda de los miembros de las organizaciones rurales, lo cual permite que sea un ejercicio incluyente y en igualdad de condiciones.

En este sentido, con base a las preguntas orientadoras elaboradas en un lenguaje sencillo, comprensible y entendible en cada uno de los componentes que integra la herramienta propuesta, se genera una retroalimentación donde los participantes socializan experiencias que hacen muy enriquecedor el trabajo en equipo, participativo y solidario.

Con relación al beneficio que las organizaciones productivas rurales perciben de este tipo de herramientas, vale la pena destacar el carácter lúdico y participativo de “Modela tu negocio fácill. En efecto se trata de una herramienta que ha sido diseñada para promover que todos los participantes aporten alrededor de la estructuración de la innovación y el modelo de negocio más adecuado según las características propias de la organización. También se resalta que una de las debilidades de organizaciones de este tipo es la falta de disciplina en el registro de actas, listados, y soportes documentales (especialmente en aquellas organizaciones de menor escala). En ese sentido, se trata de una herramienta que ha sido diseñada para promover que todos los participantes aporten alrededor de la estructuración de la innovación y el modelo de negocio más adecuado según las características propias de la organización 


\section{Conclusiones}

Las metodologías relacionadas con los modelos de negocio fueron creadas y validadas en contextos de Europa y Estados Unidos, lo anterior demanda que se realicen profundos procesos de adaptación para lograr resultados en contextos como el colombiano en general y en el departamento del Cauca en particular. Se requiere un abordaje especial en el ámbito de la innovación dado que las metodologías originales promueven la innovación tecnológica y de producto y están fuertemente sesgadas hacia la búsqueda de proyectos de innovación radical.

En este orden de ideas, una de las fortalezas de la herramienta "Modela tu negocio fácil" es la búsqueda y posterior visibilización de ejercicios piloto de innovación incremental que redunden en importantes avances en términos de innovación social.

Se destaca de la herramienta, la concepción bajo un enfoque sistémico y participativo, que propende por la inclusión de los integrantes de la Organización en su desarrollo y que permite concebir un modelo de negocio a partir de un mejor reconocimiento del entorno y del mercado, y de las capacidades técnicas y de gestión de las organizaciones rurales.

El enfoque sistémico de la herramienta fomenta la participación activa el diálogo, la interacción y el trabajo colaborativo de los miembros de la organización rural a la hora de realizar su modelo de negocio. La herramienta permite que los componentes del modelo de negocio se articulen y tengan una secuencia y a la vez interdependencia y de esta manera concebir el modelo de negocio como un todo.

En el entorno de las organizaciones rurales, es frecuente encontrar factores críticos, relacionados con su estructura organizativa, su capacidad productiva y asociatividad que no están resueltos y por lo tanto dificultan el planteamiento de un modelo de negocio innovador, por tal razón se plantea en este trabajo la existencia de algunas condiciones mínimas habilitantes para que un modelo de negocio funcione en este tipo de organizaciones. Elementos relacionados con componentes como 
Productivo, Organizativo, Asociativo y Socio clave o alianzas, son fundamentales para que las organizaciones puedan pensar en estrategias novedosas de modelos de negocio.

Se puede comprobar que a partir de las aplicaciones y validaciones con las cinco (5) organizaciones rurales del departamento del Cauca-Colombia: Corseda, Apropesca, Asorcafé, Arcauca y Rescate Cacaotero, pertenecientes a las cadenas productivas de la seda, piscicultura, cafés especiales, yuca y cacao, el modelo implementado responde a las necesidades productivas, comerciales, asociativas, organizativas, financieras y aporta una dinámica emprendedora y participativa para generar alternativas de solución con un nuevo modelo de negocio.

\section{Referencias bibliográficas}

Bertalanfy, Ludwig. (1976). Teoría general de los sistemas. México D.F: Fondo de cultura económica. Extraído de: https://cienciasyparadigmas.files.wordpress.com/2012/06/teoria-general-de-lossistemas-_-fundamentos-desarrollo-aplicacionesludwig-von-bertalanffy.pdf Consulta: $24 / 06 / 18$

Blank, Steve. (2013). The Four Steps to the Epiphany: Successful Strategies for Products that Win. (S. Blank, Ed.). Lulu Enterprises Incorporated, 2003.USA

Blank, Steve \& Dorf, Bob. (2012). The startup owner's manual. K \& S Ranch publishers.USA.

Landazury, Luis \& Ferrer, Franklin. (2016). Innovación como eje transversal de los modelos de negocio en las organizaciones, una revisión del constructo teórico. Revista Escuela de Administración de Negocios, 81, 129-148. https://doi.org/10.21158/01208160.n81.2016.1559. Consulta:25/06/18.

López, Francisco \& Castrillón, Pepe. (2007). Evolución y desarrollo de la agroindustria AI en Colombia. Revista Asuntos Económicos y Administrativos, 12, Colombia (Pp. 9-35).

Maurya, Ash. (2014). Running Lean: Cómo iterar de un plan A a un plan que funciona. (Universidad Internacional La Rioja, Ed.). O`Reilly. USA

Maurya, Ash. (2016). Scaling Lean: Mastering the key metrics for startup growth. Penguin Random House. USA.

Morales, Alfonso. (2009). Innovación social: un ámbito de interés para los servicios sociales. Córdoba. Extraído de: http://www.fundacionede.org/innovacion/docs/contenidos_innovacion/Innovaci on-Social-Servicios-Sociales.pdf. Consulta: 30/05/18. 
Osterwalder, Alexander \& Pigneur, Yves. (2010). Generación de modelos de negocio: Un manual para visionarios, revolucionarios y retadores. Deusto. USA.

Osterwalder, Alexander; Pigneur, Yves; Papadakos, Patricia; Bernarda, Gregory \& Smith, Alan. (2014). Value Proposition Design: How to Create Products and Services Customers Want. Wiley.USA

Piñeiro, David. (1985). El plenario de pequeños y medianos productores: un intento de crear un movimiento de agricultores familiares. Ed Mc Gaw Hill. México Ries, Eric. (2011). The Lean StartUp (3rd ed.). Crown. USA

Ries, Eric. (2017). The Startup Way: How Modern Companies Use Entrepreneurial Management to Transform Culture and Drive Long-Term Growth. Currency. USA.

Rodriguez, Daniel \& Riveros, Hernando. (2016). Esquemas de comercialización que facilitan la articulación de productos agrícolas con los mercados. Mc Graw Hill. México.

Vargas, Isidro \& Montoya, Iván. (2011). Análisis sistémico y viabilidad de organizaciones rurales en Colombia. In Comunidad Colombiana de Dinámica de sistemas (Ed.). $9^{\circ}$ Encuentro Colombiano de Dinámica de Sistemas (Pp. 1416). Universidad Colegio Mayor Nuestra Señora del Rosario. Colombia. 